\title{
Análisis por Elementos Finitos de Alúmina Sometida a Esfuerzos Mecánicos y Térmicos
}

\author{
A.G. Tomba Martinez, A.L. Cavalieri \\ Universidad Nacional de Mar del Plata, Instituto de Investigaciones en Ciencia y \\ Tecnología de Materiales (INTEMA), Facultad de Ingeniería, \\ Mar del Plata, Argentina
}

Received: Julho 5, 2000; Revised: December 7, 2000

\begin{abstract}
Disks of a commercial alumina were fabricated by slip casting, calcination and sintering. The surfaces were machined using $\mathrm{SiC}$ papers (120 and 320 grit) and characterized by residual stresses measurements. The mechanical strength was determined in biaxial flexure (ball on discontinuous ring). The specimens were subjected to thermal shock conditions (cooling using a high-velocity air jet) and the critical temperature differential for crack propagation was determined. The temperature and stress distributions during air impinging were calculated using a finite element method. The value of the heat transfer coefficient was estimated by fitting the calculated temperature profiles with those measured during each test. The calculated tension for the thermal shock fracture was compared with the mechanical strength, together with the fracture features in each case. The differences were explained on the basis of the calculated stress distributions.
\end{abstract}

Keywords: biaxial flexure, thermal shock, finite elements

\section{Introducción}

Los materiales cerámicos sometidos a cargas mecánicas o termomecánicas pueden fallar cuando se alcanza alguna condición crítica (criterio de fractura). En general, se asume que la respuesta de un material a uno u otro tipo de tensiones es equivalente en cuanto el proceso físico último que produce el estado de tensiones, es decir, el estiramiento de enlaces atómicos, es el mismo en ambos casos. En base a esto, cuanto mayor sea la información acerca del comportamiento mecánico de un material, mayor será la comprensión de su respuesta ante condiciones más complejas como las que involucra el choque térmico. Sin embargo, este objetivo no puede lograrse sin contar además con el valor de las tensiones térmicas generadas, para lo cual el cálculo numérico por elementos finitos resulta útil. Con este fin se han diseñado nuevos ensayos de choque térmico que satisfacen los requerimientos para estos estudios, especialmente respecto a las condiciones de borde controladas ${ }^{1-7}$. Es importante tener en cuenta que para interpretar el daño por choque térmico a la luz de la fractura en un ensayo mecánico, se requiere que ambas solicitaciones produzcan distribuciones de tensiones equivalentes.

En este trabajo se analizan comparativamente los resultados de ensayos mecánicos y de choque térmico de un

e-mail: agtomba @fi.mdp.edu.ar material de alúmina, utilizando valores de las tensiones de origen térmico obtenidos por cálculo numérico. Se incluye el análisis de la fractura en ambas solicitaciones. Los ensayos empleados, flexión biaxial y enfriamiento brusco con un jet de aire, se seleccionaron teniendo en cuenta que en ambas condiciones se genera un estado equibiaxial de tensiones sobre la superficie de la probeta. En el ensayo de choque térmico empleado las condiciones de contorno están bien establecidas permitiendo estimar valores del coeficiente de transferencia de calor superficial confiables, facilitando el cálculo de las tensiones generadas durante el ensayo.

\section{Materiales y Metodos}

\subsection{Preparación de las probetas}

Se fabricaron discos de $17,50 \pm 0,02 \mathrm{~mm}$ de radio $(R) \mathrm{y}$ 2,68 $\pm 0,02 \mathrm{~mm}$ de espesor $(t)$ por colaje de una suspensión acuosa de alúmina comercial de alta pureza (Reynolds RC-HP DBM, D $\left.50=0,35 \mu \mathrm{m}, \mathrm{S}_{\mathrm{e}}=7,3 \mathrm{~m}^{2} / \mathrm{g}\right)^{8}$. Los mismos fueron calcinados a $900{ }^{\circ} \mathrm{C}$ durante $1 \mathrm{~h}$ y luego desbastados sucesivamente con papeles de $\mathrm{SiC}$ de grados abrasivos 120 , 320 y 600 para el ajuste dimensional. El sinterizado se llevó a cabo en aire a $1600{ }^{\circ} \mathrm{C}$ durante $2 \mathrm{~h}$ (velocidad de calentamiento de $\left.2{ }^{\circ} \mathrm{C} / \mathrm{min}\right)$. Se obtuvo un material denso (99,5\% 
de la densidad teórica) de microestructura homogénea con porcentaje similar de granos equiaxiales y alargados (esbeltez $\approx 2,6$ ), con un tamaño medio de grano de 3,46 $\mu \mathrm{m}$ y muy pocos poros intragranulares. Luego del sinterizado, una de las superficies de cada disco fue pulida con papeles de $\mathrm{SiC}$ de grados abrasivos 120 y 320, sucesivamente.

La superficie pulida fue caracterizada por medidas de tensiones residuales mediante difracción de rayos $\mathrm{X}$. Se utilizó un equipo Rigaku Strainflex con radiación de $\mathrm{CrK}_{\alpha}$ y se empleó la técnica del $\operatorname{sen}^{2} \varphi$ para el cálculo de las tensiones. Los valores obtenidos para los planos de difracción $\left\{\begin{array}{lll}1 & 0 & 10\end{array}\right\}$ y $\left\{\begin{array}{lll}1 & 1 & 9\end{array}\right\}$ fueron siempre compresivos:-91,28 $\pm 10,24 \mathrm{MPa}$ y $-60,9 \pm 7,21 \mathrm{MPa}$, respectivamente.

\subsection{Ensayo mecánico}

Se determinó la resistencia a la fractura en flexión biaxial utilizando un dispositivo construido en acero especial K según la configuración de bola sobre anillo discontinuo 9 (9,75 \pm 0,02 mm de radio). Los ensayos se llevaron a cabo en una máquina universal de ensayos mecánicos hidráulica Instron modelo 8502, en control por posición a una velocidad de desplazamiento del actuador servohidráulico de $0,05 \mathrm{~mm} / \mathrm{min}$.

\subsection{Ensayo de choque térmico}

Los ensayos de choque térmico se realizaron en un equipo construido para tal fin ${ }^{9}$. Cada disco se coloca horizontalmente sobre un soporte refractario con la superficie pulida hacia arriba y con dos termocuplas en contacto con la superficie inferior en el centro y a 14,5 $\pm 0,02 \mathrm{~mm}$ del mismo. Un horno cilíndrico permite calentar el sistema a una temperatura prefijada $\left(T_{i}\right)$. Luego de estabilizar el sistema a esa temperatura durante $90 \mathrm{~min}$, se insufla un jet de aire comprimido a temperatura ambiente $\left(T_{0}=27^{\circ} \mathrm{C}\right)$ sobre el centro del disco durante $20 \mathrm{~s}$. El aire se conduce a una velocidad de $330 \mathrm{~m} / \mathrm{s}$ por un tubo de sílice de 1,79 \pm $0,02 \mathrm{~mm}$ de radio $\left(r_{0}\right)$ posicionado perpendicularmente a $3,00 \pm 0,02 \mathrm{~mm}$ sobre la superficie a ensayar.

Luego de cada ensayo se examina la probeta para detectar la aparición de fisuras. Si el examen resulta negativo el disco se somete a un nuevo ensayo, con $T_{i}$ incrementada en $10{ }^{\circ} \mathrm{C}$. Este procedimiento se repite hasta la aparición de fisuras. Usando este método se evalúa la resistencia al choque térmico $T_{C}$ (o $\Delta T_{C}=T_{C}-T_{0}$ ) definida como el valor de $T_{i}$ al cual se produce extensión de fisuras (condición de iniciación).

Durante el ensayo se registra la temperatura sobre los dos puntos de la superficie inferior del disco con termocuplas de respuesta rápida de $\mathrm{Pt}-10 \% \mathrm{Rh} / \mathrm{Pt}$ calibradas, con adquisición y almacenamiento de datos por computadora. Cuando se producen fisuras o fractura, este evento se detecta como una caída brusca en los registros de la tem- peratura, a partir de lo cual se determinan los tiempos de fractura.

El rango de valores de $T_{i}$ depende del material ensayado. Para el material de alúmina que se usó en este trabajo $T_{i}$ se varió entre $870{ }^{\circ} \mathrm{C}$ y $1030{ }^{\circ} \mathrm{C}$.

\subsection{Patrones de grietas}

Los discos fracturados en ambas condiciones, mecánica y térmica, se inspeccionaron ópticamente determinándose las características de las grietas resultantes y de las superficies de fractura. En los casos de probetas dañadas en condiciones de choque térmico en las que no se produjo fractura completa, las fisuras se revelaron por tinción con un líquido penetrante para su inspección ocular.

\subsection{Cálculo de distribuciones de temperatura y tensión}

La evolución de la temperatura y el esfuerzo en el espacio y el tiempo en los ensayos de choque térmico se calculó por el método de elementos finitos implementado en el sistema comercial LUSAS. Dada la simetría de revolución del sistema, se simuló sólo la mitad de la sección media del disco y del cemento refractario como un problema bidimensional axisimétrico. Se utilizó una malla regular de 576 elementos cuadrados parabólicos (1906 nodos), con una mayor discretización en la zona correspondiente al jet de aire. Para el cálculo se usaron valores de la literatura de las propiedades térmicas y mecánicas de la alúmina, considerando su dependencia con la temperatura ${ }^{10}$, y del cemento refractario a $200{ }^{\circ} \mathrm{C}^{11}$.

Las condiciones de contorno térmicas consideradas fueron de flujo impuesto en la cara superior, con el coeficiente de transferencia de calor superficial $(h)$ constante con la temperatura, y adiabaticidad en el resto de la frontera. Se permitió el flujo de calor por contacto entre la superficie inferior del disco y la superior del cemento refractario, con un valor estimado de la conductancia de $10^{3} \mathrm{~W} / \mathrm{m}^{2}{ }^{\circ} \mathrm{C}^{9}$.

El coeficiente de transferencia de calor superficial se estimó, como función de la distancia al centro del disco $(r)$, a través del ajuste de la variación temporal de las temperaturas obtenidas por cálculo con las registradas durante el ensayo en los puntos de la superficie inferior. Se utilizaron como curvas experimentales los promedios de los registros obtenidos a cada $T_{i}$.

El efecto de la variación en la transferencia de calor por el flujo de aire desde la zona de impacto hacia la periferia del disco se simuló con un modelo que considera un flujo turbulento a lo largo del radio, con un $h$ constante dentro de la zona de impacto del jet, un aumento del mismo en la zona de estancamiento ${ }^{12,13}$ y su posterior disminución hacia la periferia del disco, según la Ec. (A):

$$
h(r)=h_{0} \quad \text { para } r \leq r_{0}
$$




$$
\begin{array}{ll}
h(r)=\frac{h_{0}}{r} r_{0} & \text { para } r_{0}<r \leq r_{c} \\
h(r)=\frac{h_{0}}{r} r_{0} & \text { para } r_{c}<r \leq R
\end{array}
$$

donde $r_{0}$ es el radio del jet de aire, $R$ es el radio del disco y $h_{0}$ es el valor de $h$ en la zona del jet. Para $r_{C}$, que es el punto a partir del cual cambia el comportamiento de $h$, se tomó un valor de 4,74 $\pm 0,65 \mathrm{~mm}$, algo mayor a $2 r_{0}{ }^{9,12,13}$. Los parámetros de ajuste son $h_{0}$ y $h_{0}$ ' y sus valores se estimaron de acuerdo a una metodología diseñada para ese fin? .

Para el cálculo de los esfuerzos se consideró un problema de deformaciones axisimétricas. Se restringió el desplazamiento radial para los nodos ubicados sobre el eje central, tanto en el disco como en el cemento refractario. Ambos materiales se consideraron elásticos, lineales e isotrópicos.

\section{Resultados}

\subsection{Resultados experimentales}

Se emplearon 20 discos de alúmina para cada tipo de ensayo, mecánico y de choque térmico. Para los especímenes ensayados en flexión biaxial se obtuvo una resistencia mecánica de $220 \pm 66 \mathrm{MPa}$ y un módulo de Weibull de $6,9^{14}$. El valor de $\Delta T c$ medio determinado en condiciones de choque térmico resultó de $916{ }^{\circ} \mathrm{C}$, con una desviación estándar de $54{ }^{\circ} \mathrm{C}$ y los tiempos de fractura estuvieron en el rango de 3 a $15 \mathrm{~s}$.

En los discos que sufrieron fractura frente a carga mecánica se obtuvo un patrón de grietas radiales a partir de la zona central del disco. El 70\% de las probetas se rompieron en 2 partes y el resto en 3 . En general no se observaron desviaciones de los pasos de grieta mientras que las superficies de fractura fueron bastante lisas y planas, con pocos relieves. A diferencia de las probetas ensayadas en flexión biaxial, en las fracturadas en condiciones de choque térmico se observaron tanto grietas circulares como radiales. En todos los casos, la fractura parece originarse en la zona central del disco. En la mayoría de las probetas la fractura fue completa. En los casos donde las fisuras no alcanzaron la periferia del disco, éstas siempre fueron pasantes. Los pasos de las grietas resultaron bastante tortuosos y las superficies de fractura, irregulares. En general, las probetas se fracturaron en un número alto de fragmentos (entre 3 y 7 partes).

La tortuosidad y la alta fragmentación son aspectos que se asocian comúnmente a una elevada energía disponible en el momento de la iniciación de la fractura. Dadas las discrepancias observadas con la fractura mecánica, se infiere que ésta ocurre con una energía almacenada menor que la disponible en la fractura por choque térmico.

\subsection{Análisis numérico}

Para el ajuste del coeficiente de transferencia de calor superficial, y dada su dependencia con la temperatura, se seleccionaron tres valores de $T_{i}: 870{ }^{\circ} \mathrm{C}$ (bajo), $920{ }^{\circ} \mathrm{C}$ (intermedio) y $980{ }^{\circ} \mathrm{C}$ (alto), como representativos del comportamiento en todo el rango estudiado. Los valores de $h_{0}$ y $h_{0}$ ' ajustados se reportan en la Tabla 1 . Se verificó la dependencia de $h$ con $T_{i}^{9,15}$ y su valor radial máximo se localizó en $r_{C}$.

A lo largo del espesor, las menores temperaturas se producen en la superficie superior. En la Fig. 1 se muestra la variación radial de la temperatura en esta superficie para $T_{i}=920^{\circ} \mathrm{C}$ a los $10 \mathrm{~s}$ de iniciado el enfriamiento (para las otras $T_{i}$ se observa un comportamiento semejante). La temperatura presenta máximos y mínimos (éstos últimos ubicados en el centro y en $r=5,72 \mathrm{~mm}$ ) producidos por la variación radial de $h$ considerada.

La Fig. 2 corresponde a los gráficos de contorno de las componentes radial y circunferencial de las tensiones $\left(\sigma_{x} \mathrm{y}\right.$ $\left.\sigma_{z}\right)$ para $10 \mathrm{~s}$ de iniciado el enfriamiento y $920{ }^{\circ} \mathrm{C}$ de temperatura inicial. La distribución de la tensión calculada presenta máximos y mínimos locales, tanto en $\sigma_{z}$ como en $\sigma_{x}$. Este comportamiento refleja el aumento de $h$ en $r_{C}$ considerado por este modelo. La posición del máximo esfuerzo es: o bien en el punto central $\left(\sigma_{x}=\sigma_{z}\right)$ o bien a un radio $r_{M}$ de $5,72 \mathrm{~mm}\left(\sigma_{z}\right)$, en relación con los mínimos calculados en temperatura. La ocurrencia de uno u otro caso depende de la magnitud de $h_{0}$ en relación con el valor del

Tabla 1. Parámetros de ajuste.

\begin{tabular}{lcc}
\hline $\mathrm{T}_{\mathrm{i}}\left({ }^{\circ} \mathrm{C}\right)$ & $\mathrm{h}_{0}\left(\mathrm{~W} / \mathrm{m}^{\left.2{ }^{\circ} \mathrm{C}\right)}\right.$ & $\mathrm{h}_{0}{ }^{\prime}\left(\mathrm{W} / \mathrm{m}^{2 \circ} \mathrm{C}\right)$ \\
\hline 870 & 160 & 650 \\
920 & 180 & 650 \\
980 & 200 & 650 \\
\hline
\end{tabular}

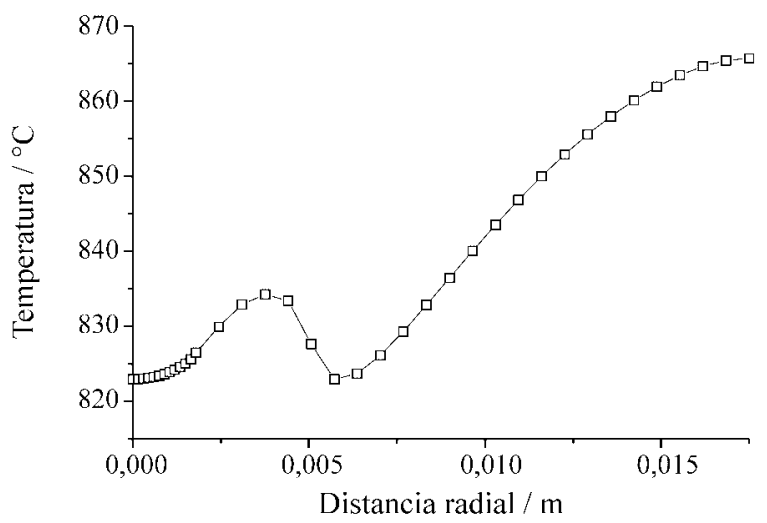

Figurz 1. Distribución radial de la temperatura sobre la superficie superior $\left(T_{i}=920^{\circ} \mathrm{C}\right.$, tiempo $\left.=10 \mathrm{~s}\right)$. 

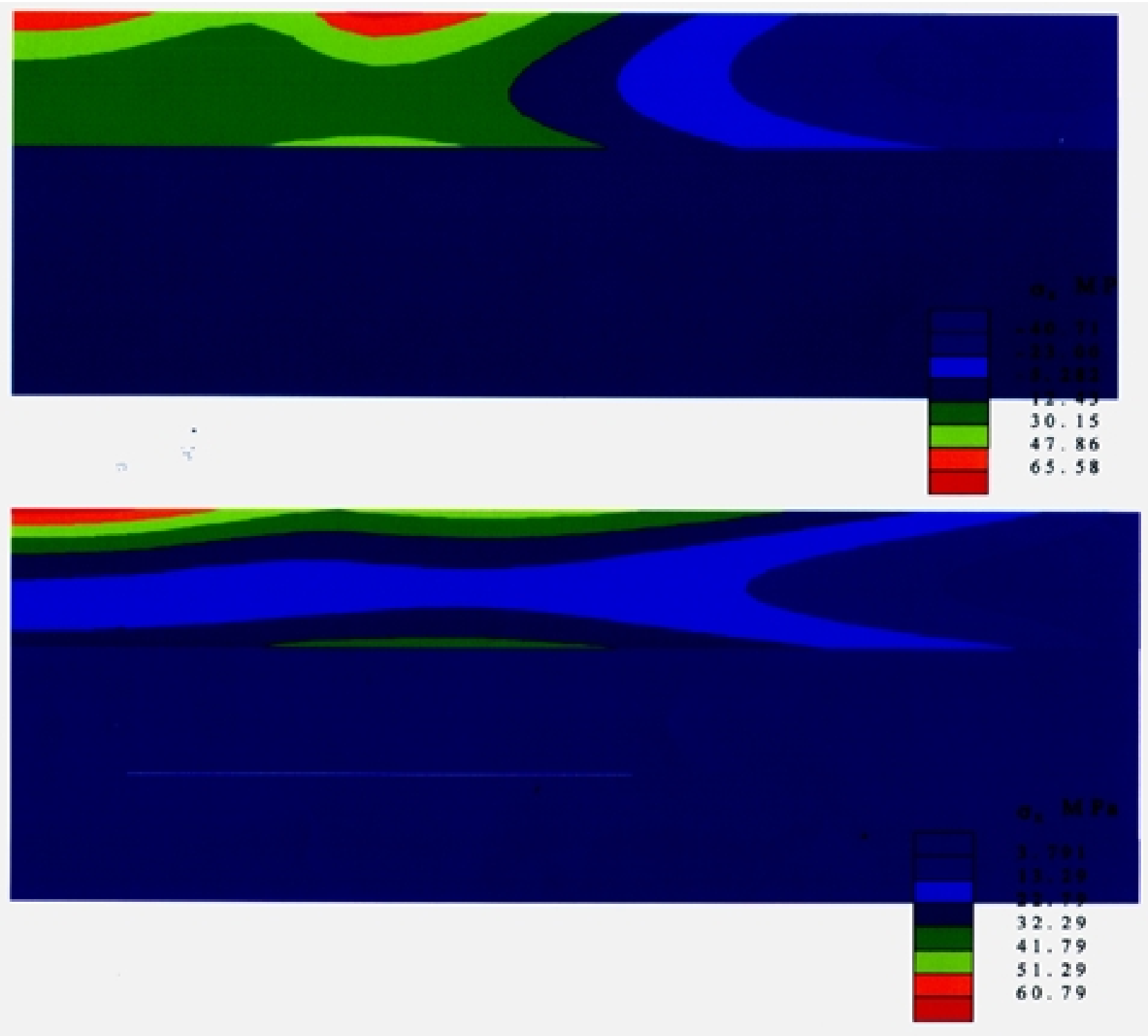

Figura 2. Gráficos de contorno de las tensiones circunferencial $\left(\sigma_{z}\right)$ y radial $\left(\sigma_{x}\right)\left(T_{i}=920^{\circ} \mathrm{C}\right.$, tiempo $\left.=10 \mathrm{~s}\right)$.

coeficiente en $r_{C}$ (función a su vez de $h_{o}$ ') y además, del tiempo de iniciado el choque térmico.

A partir del análisis de la distribución radial de la tensión se determinó la extensión de la región de equibiaxialidad en un área circular de $2 \mathrm{~mm}$ de radio. La componente circunferencial de la tensión $\left(\sigma_{z}\right)$ disminuye muy rápidamente luego de su valor máximo en $r_{M}$ hacia la periferia del disco y se vuelve compresiva a una distancia $\approx 9 r_{o}$, mientras que la componente radial en función del radio, es siempre en tracción.

En el espesor, los esfuerzos son en tracción hasta una distancia de $\approx 5,3 r_{0}$ y además, en el eje central $\sigma_{x}$ es igual a $\sigma_{z}$. Estos resultados indican la existencia de una amplia zona en tracción en el sentido del espesor (equivalente al mismo para la geometría estudiada) que sería responsable de una fractura con alta energía. Esto es característico del enfriamiento con medios de 'quenching' poco eficientes como el aire ( $h$ bajos) ${ }^{16}$. En estas condiciones, la profundidad de la capa en tracción a partir de la superficie enfriada es grande y se desarrolla lentamente, puesto que el valor del coeficiente de transferencia da tiempo para que el calor pueda fluir desde una mayor profundidad. La fractura ocurre con gran violencia, luego de un cierto tiempo de iniciado el enfriamiento, a causa de la gran cantidad de energía almacenada en una profundidad considerable del especimen.

En la Fig. 3 se observa la variación temporal del esfuerzo para $920{ }^{\circ} \mathrm{C}$ en los puntos central y $r_{M}$ sobre la superficie. Los esfuerzos calculados presentan siempre máximos a tiempos entre 2,5 y $5 \mathrm{~s}$. En la Tabla 2 se presentan los valores máximos, que en todos los casos 


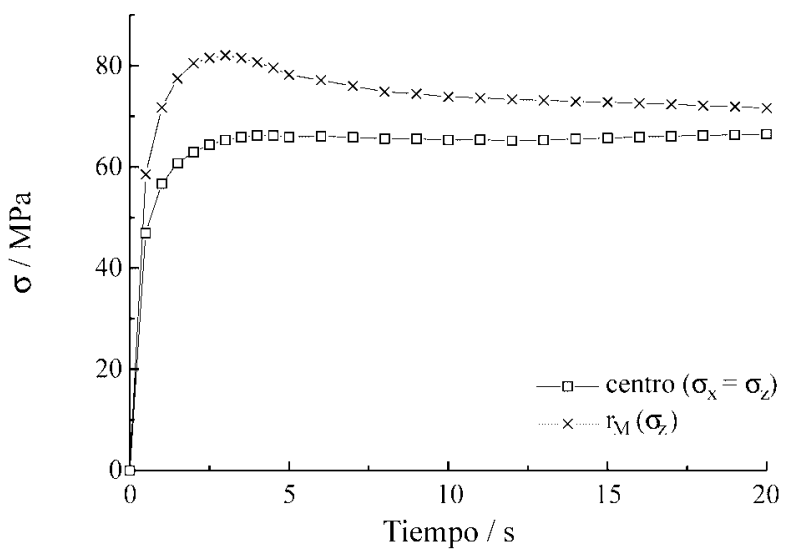

Figure 3. Variación temporal de la tensión para el centro y $r_{M}\left(\sigma_{z}\right)$ $\left(T_{i}=920^{\circ} \mathrm{C}\right)$.

Tabla 2. Valores máximos de las tensiones.

\begin{tabular}{lccc}
\hline $\mathrm{T}_{\mathrm{i}}\left({ }^{\circ} \mathrm{C}\right)$ & $\sigma_{\mathrm{z}}(\mathrm{MPa})$ & Posición & tiempo $(\mathrm{s})$ \\
\hline $870^{\circ} \mathrm{C}$ & 77 & $r_{M}$ & 3,6 \\
$920^{\circ} \mathrm{C}$ & 81,5 & $r_{M}$ & 3,0 \\
$980^{\circ} \mathrm{C}$ & 89 & $r_{M}$ & 3,4 \\
\hline
\end{tabular}

ocurren en $\sigma_{z}$, junto con el tiempo y la posición correspondientes a las tres temperaturas iniciales consideradas.

\section{Discusión}

Los patrones de grietas obtenidos tanto para los discos fracturados mecánicamente como para los sometidos a condiciones de choque térmico son consistentes con la distribución equibiaxial de tensiones en ambos tipos de configuración de carga.

En la flexión biaxial de bola sobre anillo discontinuo, la magnitud de las tensiones circunferencial y radial en la zona central de la superficie de la probeta es máxima e igual para ambas componentes. Los valores máximos se mantienen constantes hasta un radio de aproximadamente $t / 3^{17}$. Esto concuerda con la presencia de grietas radiales que se iniciarían en la zona central.

La aparición de fisuras radiales y circulares en choque térmico se justifica, respectivamente, por el cálculo de tensiones circunferenciales y radiales de magnitudes elevadas y semejantes. La presencia de grietas circulares fuera de la zona de impacto del jet en algunas probetas puede relacionarse con los valores máximos de las tensiones obtenidos más allá de esta región. Por otra parte, las fisuras no siempre alcanzan la periferia y esto puede asociarse a que la tensión circunferencial $\left(\sigma_{z}\right)$ se hace compresiva, contribuyendo al arresto de las grietas. Además, siempre traspasan el espesor en concordancia con el hecho de que en una parte importante de la probeta, los esfuerzos calculados a lo largo del espesor resultaron sólo en tracción.
Los tiempos a los que aparecen los máximos de las tensiones calculadas en condiciones de choque térmico están dentro del rango de los tiempos de fractura determinados experimentalmente (3-15 s).

Asumiendo que la fractura por esfuerzos de origen térmico se produce al mismo valor crítico de tensiones que la que ocurre por esfuerzos mecánicos (para configuraciones equivalentes de esfuerzos como es este el caso), al comparar las tensiones de fractura medidas en flexión biaxial $\left(\sigma_{F}\right)$ con las tensiones calculadas a los tiempos de fractura experimentales en los ensayos de choque térmico (Tabla 2), las últimas resultaron más bajas.

Una posible explicación para esta diferencia puede darse, por un lado, considerando la existencia de esfuerzos residuales compresivos originados por el tratamiento superficial de pulido de los discos. Tales esfuerzos pueden actuar modificando la distribución de tensiones en una solicitación mecánica o termomecánica, generalmente aumentando la resistencia mecánica ${ }^{18}$. En los ensayos de choque térmico, cada probeta se trata a temperaturas relativamente elevadas durante tiempos prolongados, no pudiéndose descartar que en estas condiciones se produzca una relajación de las tensiones residuales con modificación de la resistencia del material a soportar esfuerzos respecto del ensayo mecánico.

Otra razón para esta discrepancia se puede encontrar en el análisis de la probabilidad de hallar un defecto crítico en la zona de equibiaxialidad. En el ensayo de choque térmico, esta región se extiende a un área de $2 \mathrm{~mm}$ de radio, cinco veces mayor que en el ensayo mecánico $(0,89 \mathrm{~mm} \approx t / 3)$, a la que se suma una zona adicional que circunda a $r_{M}$ sometida a altos esfuerzos. En consecuencia, en la solicitación térmica la probabilidad sería más alta y los valores de tensión de fractura resultarían menores.

La mayor cantidad de energía disponible en el momento de la fractura en las probetas sometidas a condiciones de choque térmico respecto de las solicitadas mecánicamente, inferida a partir de la tortuosidad y mayor fragmentación de las primeras, es consistente con los resultados numéricos obtenidos. Durante el enfriamiento brusco el espesor del disco está completamente en tracción hasta una distancia de $\approx 5,3 r_{0}$ mientras que en los ensayos mecánicos en flexión biaxial siempre existe un plano (fibra neutra) a partir del cual la tensión se vuelve compresiva, incluso en la zona central de la probeta. En consecuencia, la energía almacenada en el momento de la fractura sería siempre mayor en condiciones de choque térmico conduciendo a una fractura más violenta con grietas que sufren múltiples desviaciones y rompiéndose la probeta en varias partes.

\section{Conclusiones}

Se encontraron diferencias entre los resultados experimentales, valores de resistencia y características de la frac- 
tura, obtenidos en los ensayos mecánicos y los calculados a partir de los $\Delta T_{C}$ en los ensayos de choque térmico de discos de alúmina. Estas se explicaron teniendo en cuenta las distribuciones de tensiones calculadas. Si bien se tienen distribuciones equibiaxiales de tensiones, no resultan idénticas en la superficie ensayada del disco y las diferencias se hacen más marcadas a lo largo del espesor.

\section{Referencias}

1. Faber, K.T.; Huang, M.D.; Evans, A.G. J. Am. Ceram. Soc., v. 64, p. 296-301, 1981.

2. Niihara, K.; Singh, J.P.; Hasselman, D.P.H. J. Mat. Sci., v. 17, p. 2553-2559, 1982.

3. Brockenbrough, J.R.; Edgar Forsythe, L.; Rolf, R.L. J. Am. Ceram. Soc., v. 69, p. 634-637, 1986.

4. Wei, G.C.; Walsh, J. J. Am. Ceram. Soc., v. 72, p. 1286-1289, 1989.

5. Schneider, G.A.; Petzow, G. J. Am. Ceram. Soc., v. 74, p. 98-102, 1991.

6. Rogers, W.P.; Emery, A.F. J. Mat. Sci., v. 27, p. 146-152, 1992.

7. Awaji, H.; Oguwa, M.; Sato, S. Third Euro-Ceramics v. 3, Durán, P.; Fernández, J.F., eds., Faenza Editrice Iberica, S.L., Spain, p. 883-888, 1993.
8. Urretazvizcaya, G. Ph.D. Thesis, Univ. Nac. Mar del Plata, 1995.

9. Tomba Martinez, A.G. Ph.D. Thesis, Univ. Nac. Mar del Plata, 1998.

10. Gitzen, W.H. Alumina as ceramic material, The American Ceramic Society, Columbus, 1970.

11. Fisher, R.E. Advances in refractories technology, Ceramic Transactions, v. 4, The American Ceramic Society, 1988.

12. Poulson, B. Corros. Sci., v. 23, p. 391-430, 1983.

13. Cooper, D.; Jackson, D.C.; Launder, B.E.; Liao, G.X. Int. J. Heatt Mass Transfer, v. 36, n. 10, p. 2675-84, 1993.

14. Tomba Martinez, A.G.; Cavalieri, A.L. Mat. Res. Bull., v. 35, p. 1077-1085, 2000.

15. Tomba Martinez, A.G.; Cavalieri, A.L. Mat. Lett., v. 42, p. 240-245, 2000.

16. Frechette,V.D. Failure analysis of brittle materials, Advances in Ceramics, v. 28., The American Ceramic Society, Ohio, 1990.

17. Shetty, D.K.; Rosenfield, A.R.; McGuire, P.; Bansal, G.K.; Duckworth, W.H. Ceram.Bull., v. 59, n. 12, p.1193-1197, 1980.

18. Esposito, L.; Tucci, A.; Andalo, G. J. Eur. Ceram. Soc., v. 17, p. 479-486, 1997. 